\title{
Calcific periarthritis of the elbow presenting as acute tennis elbow
}

${ }^{1} \mathrm{~F}$ Jawad, ${ }^{2}$ ASM Jawad

${ }^{1}$ Core Medical Trainee Year 2; ${ }^{2}$ Consultant, Rheumatology Department, The Royal London Hospital, London, UK

ABSTRACT A 28-year-old woman presented with sudden acute lateral epicondylitis. There was no history of preceding trauma or repetitive use of the arm. Because of the acute onset and signs of acute inflammation, an X-ray was arranged. The X-ray showed a hyperdense calcified elongated globule distal to the lateral epicondyle. A diagnosis of calcific periarthritis (calcium apatite) of the elbow was made. Calcific periarthritis has rarely been reported as a cause of acute elbow pain.

KEYWORDS Epicondylitis, tennis elbow, calcific periarthritis, calcium apatite crystals
Correspondence to ASM Jawad Rheumatology Department The Royal London Hospital London EI 4DG, UK

tel. +44 (0)208 2238408

e-mail

alismjawadI@hotmail.com

DECLARATION OF INTERESTS No conflicts of interest declared.

\section{CASE REPORT}

A 28-year-old, right-handed woman presented to the accident and emergency department with sudden, severe pain on the lateral aspect of the right elbow of one-day duration. The patient woke up with the pain. There was no preceding trauma or any other past history of note. The pain radiated along the extensor compartment to the dorsum of the wrist and hand. Using the right hand and particularly making a fist aggravated the pain. There was severe tenderness and moderate redness over the lateral epicondyle. Because of the pain, the patient found it difficult to fully extend the elbow. She was prescribed paracetamol and codeine for the pain. There was no improvement after two days and she was seen in the rheumatology department. The clinical findings were unchanged. Because of lack of full extension of the elbow, an X-ray was arranged.

The X-ray (Figure I) showed a hyperdense calcified elongated globule distal to the lateral epicondyle. A diagnosis of calcific periarthritis (calcium apatite) of the elbow was made. An attempt to aspirate the calcium deposit under ultrasound with a wide bore needle was attempted but proved unsuccessful, likely because the calcium deposit was in a paste-like form during the acute phase. There was no effusion in the elbow. Ten milligrams of methylprednisolone with lidocaine were infiltrated around the calcification. The pain settled after 24 hours. Two weeks later, the patient remained symptom-free with full range of movement in the elbow. Four weeks later, a repeat of the X-ray showed fading of the calcification (Figure 2).

\section{DISCUSSION}

Lateral elbow pain may originate from the lateral epicondyle, the radiohumeral joint, or be referred from the shoulder or neck. The pain of lateral epicondylitis

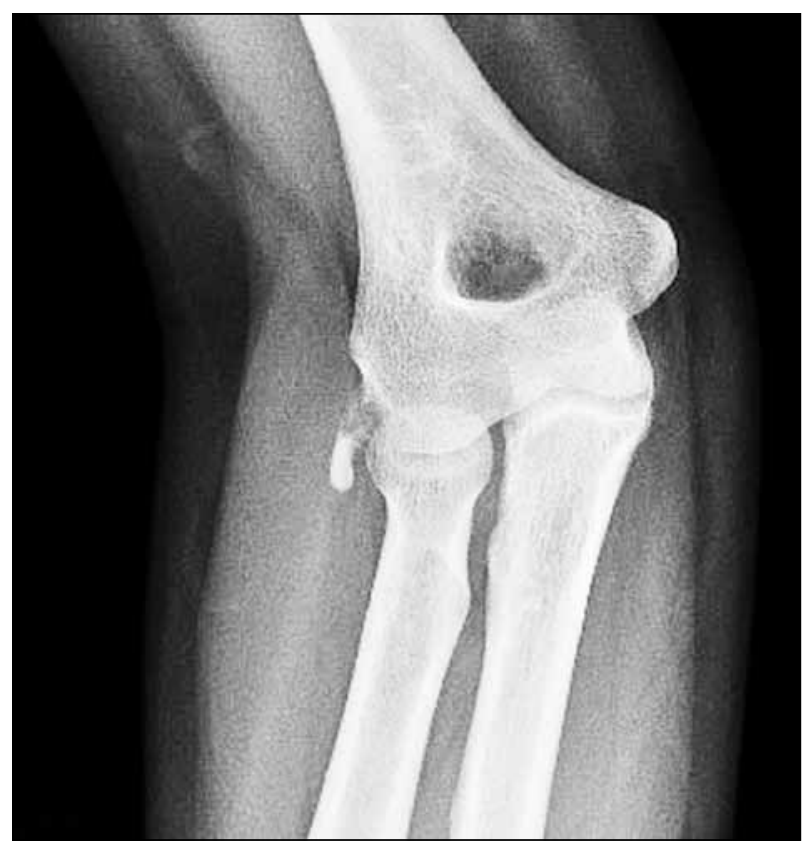

FIGURE | X-ray of the right elbow showing a hyper dense elongated calcified globule distal to the lateral epicondyle..

(tennis elbow) is typically well localised and is aggravated by activity that contracts the wrist extensors, including repetitive use of the forearm and wrist and shaking hands. Pain arising from the elbow joint is located slightly more posterior to the epicondyle along the joint line, and is easily differentiated from the pain of lateral epicondylitis by limiting range of movement of the elbow. An elbow effusion will limit extension. Referred elbow pain is suggested by its vagueness, absence of increased pain with elbow movement, and aggravation of the pain with movement of the shoulder or neck. Repetitive movements involving eccentric motion, in which the muscle-tendon unit is lengthened while contracting, may increase susceptibility to injury.' Bone marrow oedema of the lateral epicondyle (osteitis) has been reported as a 


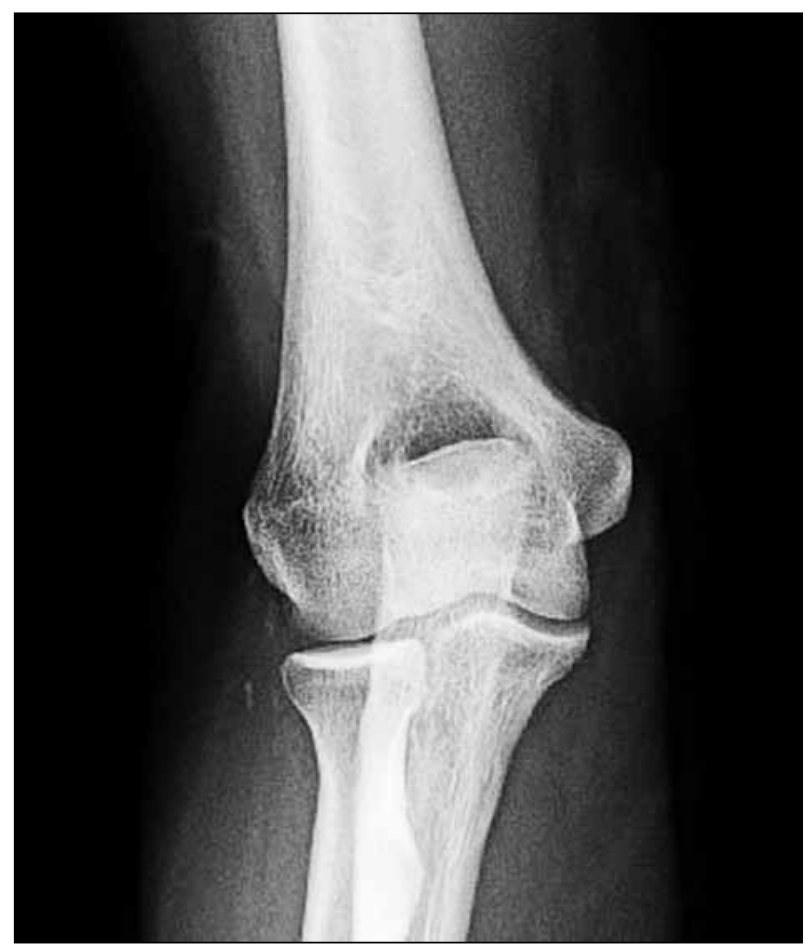

FIGURE 2 A repeat of the X-ray of the right elbow (four weeks later) showing fading of the calcification distal to the lateral epicondyle.
In the majority of cases the diagnosis of lateral epicondylitis can be made on clinical grounds. Calcific periarthritis as a cause of elbow pain is very rare $^{3-5}$ but it should be suspected and a plain X-ray requested if a patient presents with very acute epicondylitis without any preceding history of trauma or sudden overuse of the upper limb. The condition responds to a local corticosteroids injection, non-steroidal anti-inflammatory drugs or colchicine. ${ }^{3,4}$ The calcification usually resolves after a few weeks.

\section{KEY POINTS}

- In the majority of cases the diagnosis of lateral epicondylitis can be made on clinical grounds.

- Calcific periarthritis should be suspected and a plain $X$-ray requested if a patient presents with very acute epicondylitis without any preceding history of trauma or sudden overuse of the upper limb.

- The condition responds to a local corticosteroids injection, non-steroidal anti-inflammatory drugs or colchicine.

- The calcification usually resolves after a few weeks.

possible underling cause of tennis elbow. ${ }^{2}$ Acute calcific periarthritis, caused by deposition of calcium apatite crystals has been more commonly reported in the shoulder, less so in the wrist or hand but very rarely as a cause of acute elbow pain..$^{3-5}$ The aetiology and pathogenesis of acute calcific periarthritis is not clear but preceding local trauma or systemic calcium or phosphate abnormalities can contribute.

\section{REFERENCES}

I Newham DJ, Jones DA, Clarkson PM. Repeated high-force eccentric exercise: effects on muscle pain and damage.J Appl Physiol I985; 63:I38I6.

2 Lee R, Al-Adhoubi NK, Jawad ASM. Bone marrow oedema of the radius presenting as tennis elbow. Rheumatology 20I3; 52:234. http://dx.doi. org/l0.1093/rheumatology/kes290

3 Yosipovitch G, Yosipovitch Z. Acute calcific periarthritis of the hand and elbows in women: a study and review of the literature.J Rheumatol 1993; 20:1533-8.

4 Doumas C,Vazirani RM, Clifford PD et al.Acute calcific periarthritis of the hand and wrist: a series and review of the literature. Emerg Radiol 2007; I4:199-203. http://dx.doi.org/I0.1007/s I0I40-007-0626-9

5 Johnson GS, Guly HR.Acute calcific periarthritis outside the shoulder: a frequently misdiagnosed condition. J Accid Emerg Med I994; I I:I98200. http://dx.doi.org/I0.II36/emj.II.3.198 